\title{
Impact of the COVID-19 pandemic on breast cancer diagnosis
}

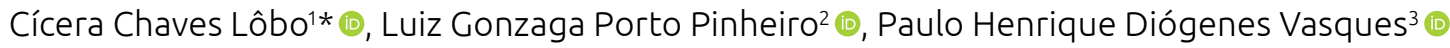

\section{ABSTRACT}

Introduction: In 2020, a total of 2,510 new cases of breast cancer were estimated in Ceará State, 14\% above the figures of 2019. In the context of the COVID-19 pandemic, postponing screening and assessing the risks and benefits of elective procedures was needed, rescheduled until after their control. Objective: We sought to identify the impact of the COVID-19 pandemic in the care of a Reference Service for Breast Cancer Diagnosis. Methods: Time series study, with analysis of the production of the consultations carried out from March to June of the current year in a service located in Fortaleza City, Ceará State. Results: There was a reduction of up to $84 \%$ in the services offered, with emphasis on mammography and ultrasound procedures, with 95 and $100 \%$, respectively. The diagnosis of new cases and the performance of surgeries reduced by up to 60 and $56 \%$, respectively. The months with the greatest impact were April and May, with a progressive resumption in June. Conclusion: The study evaluated a reference service of relevance in the state reality. Considering that many cases are identified during screening, postponing mammograms contributed to a delayed diagnosis. The findings are believed to pose severe consequences, considering the annual increase in the incidence of the disease, the low screening coverage, the high number of cases in advanced staging, the ascending mortality, and the low supply of diagnostic services. Diverting attention exclusively to the pandemic represents a worldwide challenge, but cancer is an important cause of morbidity and mortality, and cannot be neglected. There is concern that delaying screening, diagnosis, and treatment of breast cancer may cost more lives than COVID-19 itself. Post-pandemic requires planning to promote harm reduction resulting from the delay in the diagnosis and treatment of the repressed demand, in a disaggregated and overloaded system.

KEYWORDS: breast neoplasms; health services; early detection of cancer; mass screening; coronavirus infection.

\section{INTRODUCTION}

Global cancer estimates were 18.1 million new cases and 9.6 million cancer deaths in 2018. Breast cancer is recognized as the most prevalent type in the female population worldwide, except in East Africa, with 2,088,049 new cases. It represents $24.2 \%$ of the total number of cancer cases in women, with a $15.0 \%$ mortality rate and an upward estimate of 3,059,829 cases in $2040 .^{1}$

For 2020, approximately 625,000 cancer diagnoses were forecast in Brazil. Except for non-melanoma skin tumors, breast cancer is the most common type in the female population, with 66,280 new cases, $29.7 \%$ of the total cases, and an estimated risk of 61.61 cases per 100,000 women. In Ceará State, the estimate is 2,510 new cases, with approximately half of the cases in the capital. These national figures are roughly $10 \%$ higher than the previous estimate, and being above 50 is considered the most significant risk factor. The estimated increase for the state is approximately $14 \% .2,3$

Such data are like those of high-income countries, such as the United States, in which cancer also represents $30 \%$ of all new cases. But unlike those countries, in which mortality has declined in recent decades, Brazilian mortality rates still show an upward curve. ${ }^{3,4}$ Late diagnosis predominates in

\footnotetext{
${ }^{1}$ Hospital Universitário Walter Cantídio, Universidade Federal do Ceará - Fortaleza (CE), Brazil.

2Universidade Federal do Ceará - Fortaleza (CE), Brazil.

${ }^{3}$ Secretaria Municipal da Saúde - Fortaleza (CE), Brazil.

*Corresponding author: ciceralobo@bol.com.br

Conflict of interests: nothing to declare.

Received on: 09/09/2020. Accepted on: 09/27/2020
} 
$80 \%$ of cases, to the detriment of $40 \%$ in the American population. ${ }^{5}$ Another divergence is the high percentage of diagnoses in young women in the Brazilian population, outside the screening target. ${ }^{6}$

Coronavirus disease 2019 (COVID-19), caused by SARS-CoV-2, which first appeared in December 2019 in Wuhan, China, was declared as a pandemic by the World Health Organization (WHO) on March 11th, 2020. It imposed urgent and aggressive measures by worldwide national health services for the threat posed by this virus, which is highly infectious and has the potential to evolve into serious and fatal conditions, especially in older adults, immunosuppressed people, and patients with some chronic diseases. Some recommendations are promoting social distancing, detecting cases, tracking contacts, testing and treating patients in a timely manner.?

Guided by WHO recommendations, the National Cancer Institute (INCA) issued a technical note recommending that professionals advise people not to seek health services for screening mammograms, thus postponing consultations and exams until restrictions are reduced. ${ }^{8}$ The imposed measures led many patients to a temporary gap in care, considering the status of the case (risk of death), made by the team, and should be documented in the medical record. ${ }^{9}$ This recommendation is in line with that recommended in the rest of the world..$^{10-12}$

Some preliminary recommendations were postponing or continuing cancer treatment during the pandemic, based mainly on the categorization of patients at low, moderate, or high risk of disease progression without antineoplastic treatment. For some extremely aggressive tumor types, timely diagnosis and treatment is required. For others, including breast cancer, the delay in therapeutic interventions can be considered, based on the status of each case. This change may not affect the outcome in the long run, whereas potential exposure to the virus can be risky or even fatal. These recommendations can be applied with caution in current clinical practice until evidence-based guidelines are available. However, postponing breast cancer screening, diagnosis, and treatment is likely to cost more patients' lives than COVID-19 itself. ${ }^{10,11,13}$

At a time when knowledge and information emerge almost concomitantly, we sought to identify the impact of the COVID-19 pandemic in the care of a Reference Service for Breast Cancer Diagnosis.

\section{METHODS}

This is a trend analysis, or time series, carried out in July 2020 in an SDM located in the city of Fortaleza City, Ceará State. It was chosen for its role in the diagnosis and treatment of breast cancer since the early 2000s, becoming the first SDM within Ceará State in 2016. The efforts made since then have multiplied over the following years, consolidating their impact on the state network of the Brazilian Unified Health System (Sistema Unico de
Saúde - SUS). Currently, the service provides specialized medical consultation, mammography, breast ultrasound, breast puncture (with core biopsy and fine needle), breast biopsy (in the open, guided by mammography and ultrasound), and nodule excision. The result of these efforts culminates in $8 \%$ of the diagnoses of Cearás territory in a single service in the historical series of recent years.

Data were collected on the production of consultations carried out from March to June 2020, arranged in the database of the Outpatient Information System (Sistema de Informação Ambulatorial-SIA/SUS), the Hospital Information System (Sistema de Informações Hospitalares - SIH/SUS), and the Integrator of the Hospital Cancer Registry (Integrador do Registro Hospitalar de Câncer-RHC). There was univariate statistical analysis, with calculation of the average number of visits, diagnostic procedures, new cases and surgeries (mastectomies/quadrantectomies) in the previous year, 2019, and comparison with production during the pandemic period. Ethical aspects were respected for collection in open databases.

\section{RESULTS}

Considering the year of 2019 as a reference, the average number of visits was 1,411 per month, namely: specialized medical consultation, mammography, breast ultrasound, breast puncture (with core biopsy and fine needle), breast biopsy (in the open, guided by mammography and ultrasound), and nodule excision. The most frequent procedure is mammography, with an average of 781 per month. Breast cancer presents a monthly average of 15 new cases and performs an average of 18 surgeries (mastectomies).

After the COVID-19 pandemic started, March suffered a small impact, remaining on average, but there was an abrupt reduction in April, reaching a decrease of $84 \%$ in May, as shown

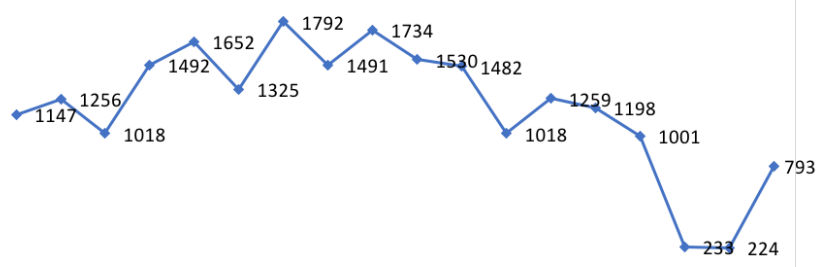

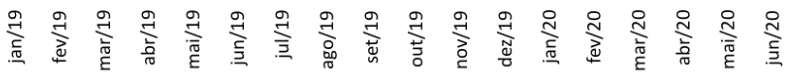

Source: Outpatient Information System (Sistema de Informação Ambulatorial - SIA/SUS).

Graph 1. Services provided in Reference Service for Breast Cancer Diagnosis (Serviço de Referência para Diagnóstico de Câncer de Mama-SDM) of the Oncology Education and Studies Group (Grupo de Educação e Estudos Oncológicos - GEEON) from January 2019 to June 2020, Fortaleza City - Ceará State, July 2020. 
in Graph 1. In mid-June, with stabilization in cases and mortality, care gradually resumed, totaling $44 \%$ of the monthly average in June.

The most frequent procedures were mammography and specialized medical consultation. However, mammography and breast ultrasound presented the greatest impact resulting from the pandemic, with a reduction of 95 and $100 \%$, respectively, as shown in Graph 2.

At first, the result of reduction in visits had a slight impact on the number of new cases diagnosed and surgical procedures, keeping them at the historical average. After the second month, there was a reduction of 60 and $56 \%$, respectively, as shown in Graph 3.

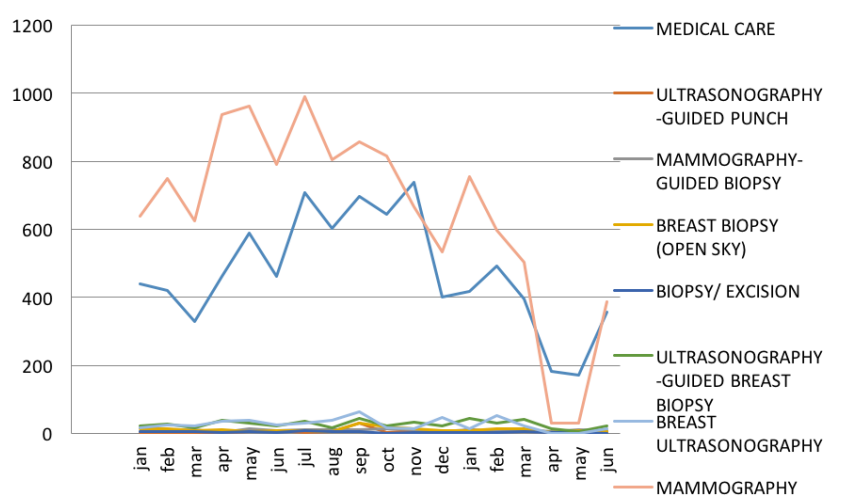

Source: Outpatient Information System (Sistema de Informação Ambulatorial-SIA/SUS).

Graph 2. Procedures performed in Reference Service for Breast Cancer Diagnosis (Oncology Education and Studies Group (Grupo de Educação e Estudos Oncológicos - GEEON) from January/ 2019 to June/2020, Fortaleza City, Ceará State, July/2020.

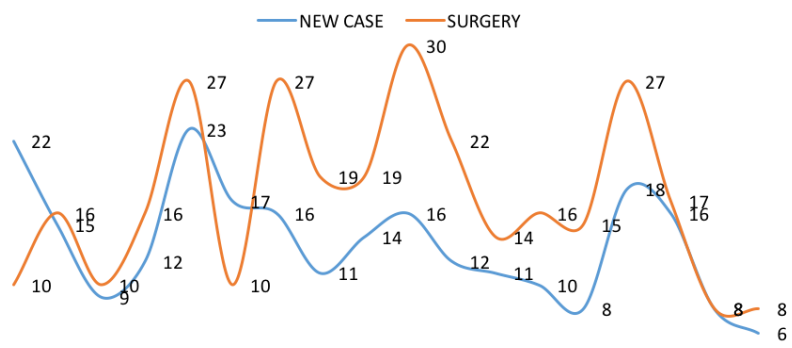

jan feb mar apr may jun jul aug sep oct nov dec jan feb mar apr may jun Source: Hospital Information System (Sistema de Informações Hospitalares - SIH/SUS) and Integrator of the Hospital Cancer Registry (Integrador do Registro Hospitalar de Câncer - RHC).

Graph 3. New cases of breast cancer and procedures performed in Reference Service for Breast Cancer Diagnosis (Serviço de Referência para Diagnóstico de Câncer de Mama-SDM) of the Oncology Education and Studies Group (Grupo de Educação e Estudos Oncológicos - GEEON) from January/ 2019 to June/2020, Fortaleza City, Ceará State, July/2020.

\section{DISCUSSION}

The best way to conduct care in early diagnosis and in the screening of breast cancer during the pandemic is uncertain, but the guidelines recommend changing our practice, which makes it difficult not to succumb to the distracting effect in the provision of care. ${ }^{8,13-15}$

Population aging has increased the overall incidence of cancer. In emerging countries, there is an epidemiological transition, a phase in which the most common types are no longer predominantly associated with inflammatory and infectious causes and are now caused by harmful lifestyles. In low and middle-income countries, late diagnosis predominates. ${ }^{16}$

Despite efforts, there will be more challenges in the future as a result of changes in standard practice after society has recovered from the COVID-19 pandemic. ${ }^{12,14,17}$ For now, focus should be placed on the immediacy of protecting cancer patients in the best possible way. ${ }^{9}$

The current COVID-19 pandemic is unprecedented, and its numbers continue increasing, but the world is preparing for a gradual resumption, including in healthcare services, limiting patient exposure. These changes will inevitably have adverse consequences in the breast cancer diagnosis, in the treatment and in the survival of patients..$^{10-12}$

Before the COVID-19 pandemic, the estimate of new cases of breast cancer for 2020 already presented a challenging scenario for health managers, considering that, year after year, morbidity and mortality figures show an upward curve, despite the implementation of health equipment and efforts to expand access to the network. The increase of approximately $14 \%$ in new cases compared to the 2018-2019 biennium, added to most diagnoses with advanced stage, reflects the severity of the condition and the absence of an effective public policy capable of controlling the disease, which increasingly affects women at ages outside the tracking range, therefore outside the target of public health policies. ${ }^{2,3,5,6}$

Another aspect to be considered is the impact of breast cancer on employment and income, increasing the percentage of the SUS-dependent population, which in March 2020 was already approximately $85 \%$ of the more than 9 million people from Ceará State. Health in Brazil has universal public funding with SUS, besides private funding/supplementary health. A third category that has recently emerged are popular clinics, which offer specialized consultations and complementary exams, targeted by patients who do not have the resources to access private/supplementary health and who do not perform procedures in SUS, highlighting herein the tracking mammography.

In the face of an erratic system and a population of knowledge and practices little used to early detect breast cancer, opportunistic screening is the moment with the possibility to identify suspicious lesions, in a system with little offer of services and saturated as to diagnostic complementation. ${ }^{16}$ 
The Oncology Education and Studies Group (Grupo de Educação e Estudos Oncológicos - GEEON) works as a differential in the state health network, exclusively serving SUS users, both local and reference. It accounts for $8 \%$ of the state's annual diagnoses, result of great efforts to ensure the expansion of the service offered. However, with the COVID-19 pandemic hitting the national territory in March, postponing consultations of patients in clinical conditions to wait, as well as mammographic screening was needed., ${ }^{89}$

Despite the reorganization of the services offered to adapt them to the sanitary conditions imposed at this time and the growing demand for new cases, the service showed a maximum reduction of $84 \%$ in the procedures offered. ${ }^{8,9,15}$ The months of April and May suffered the greatest impact, considering that the capital was in a state of collapse in this period. In June, in view of the stabilization and flexibility of isolation rules, consultations gradually returned. Even though, there is still high absenteeism, which is possibly the result of population's fear of being exposed to the virus and urban violence, especially in public transport, which has not yet had $100 \%$ of its fleet restored.

The $60 \%$ drop in the identification of new cases reflects, among other characteristics, the erratic, routine diagnosis during opportunistic screening. ${ }^{17}$ This achievement contributes to worsening the staging of new cases and, consequently, the survival of patients. ${ }^{16}$ Consequently, there was also a $56 \%$ reduction in surgeries performed.

Both delayed diagnosis and treatment need to be reprogrammed, but always considering the screening and prioritization of suspicious lesions, especially palpable lesions, as well as the investigation of the findings of BI-RADS ${ }^{18}$ categories 0,4 , and 5 .

It is not yet possible to measure the full impact of the reduction in care in the diagnosis of the disease, being the responsibility of each service the monitoring of its indicators at the first moment, considering that public health management bodies are still concerned with the disease internalization.
Bringing patient navigation to this moment is a strategy that must be considered by managers, articulating the patient and the network with social distance, using telephone contact and text messages. ${ }^{5}$

The State Committee and the Municipal Breast Cancer Committee of Fortaleza City have the mission of articulating the health system, the civil society, and class entities to minimize the "distraction effect", especially on the eve of Pink October, date traditionally used in Brazil to disseminate content, raise awareness, and expand breast cancer screening.

\section{CONCLUSION}

The findings represent a cause of great concern for the scenario after controlling the pandemic, given the increase in incidence, the low screening coverage, the high number of cases in advanced staging, the ascending mortality, the low supply of diagnostic services, aggravated by a reduction of up to $60 \%$ in diagnosis and up to $56 \%$ in surgical treatment in an SDM.

The assistance network is already saturated in most services, and the demand, which was repressed from March to June, will need to strengthen the offer to face the challenge of diagnosis and treatment in a timely manner, otherwise it will result in an even greater impact on mortality.

\section{AUTHORS' CONTRIBUTION}

C.L.: Conceptualization, investigation, methodology, validation, data curation, formal analysis, investigation, writing - original draft, writing - review \& editing.

L.P.: Conceptualization, methodology, project administration, supervision, validation, writing - review \& editing.

P.V.: Conceptualization, investigation, validation, formal analysis, investigation, writing - original draft, writing - review \& editing.

\section{REFERENCES}

1. Ferlay J, Colombert M, Soerjomataram I, Mathers C, Parkin DM, Piñeros M, et al. Estimating the global cancer incidence and mortality in 2018: GLOBOCAN sources and methods. Int J Cancer. 2019;144(8):1941-53. https://doi.org/10.1002/ijc.31937

2. Instituto Nacional de Câncer José Alencar Gomes da Silva (INCA). Estimativa 2018: incidência de câncer no Brasil. Rio de Janeiro: INCA; 2018.

3. Instituto Nacional de Câncer José Alencar Gomes da Silva (INCA). Estimativa 2020: incidência de câncer no Brasil. Rio de Janeiro: INCA; 2019.

4. American Cancer Society (ACS). Cancer Facts \& Figures 2020. Atlanta: American American Cancer Society; 2020.

5. Gioia S, Brigagão L, Torres C, Lima A, Medeiros M. The implementation of patient navigation to improve mammography coverage and access to breast cancer in Rio de Janeiro. Mastology. 2019;29(4):186-92. https://doi.org/10.29289 /25945394201920190006

6. Franzoi MA, Rosa DD, Zaffaroni F, Werutsky G, Simon S, Bines J, et al. Advanced State at Diagnosisand Worse Clinicopathologic Features in Young Women with Breast Cancer in Brazil: a Subanalysis of the AMAZONA III Study. J Global Oncology. 2019;(5):1-10. http://doi.org/10.1200/JGO.19.00263

7. World Health Organization. WHO Director-General's opening remarks at the media briefing on COVID-19 [Internet]. World Health Organization; 2020 [cited on Jun 25, 2020]. Available at: https://www.who.int/dg/speeches/detail/who-directorgeneral-s-opening-remarks-at-the-media-briefing-on-covid19---11-march-2020 
8. Instituto Nacional de Câncer José Alencar Gomes da Silva (INCA). Nota Técnica - DIDEPRE/CONPREV/INCA 30/3/2020. Detecção precoce de câncer durante a pandemia de Covid-19. Rio de Janeiro: INCA; 2020.

9. American College of Surgeons. COVID-19 Guidelines for Triage of Breast Cancer Patients [Internet]. American College of Surgeons; 2020 [cited on Jun 25, 2020]. Available at: https:// www.facs.org/covid-19/clinical-guidance/elective-case/ breast-cancer

10. Haar JV, Hoes LR, Coles CE, Seamon K, Fröhling S, Jäger D, et al. Caring for patients with cancer in the COVID-19 era. Nat Med. 2020;26:665-71. https://doi.org/10.1038/s41591-020-0874-8

11. Tang LV, Hu Y. Poor clinical outcomes for patients with cancer during the COVID-19 pandemic. Lancet Oncol. 2020;21(7):8624. https://doi.org/10.1016/S1470-2045(20)30311-9

12. El-Shakankery KH, Kefas J, Crusz SM. Caring for our cancer patients in the wake of COVID-19. Brit J Cancer. 2020;123:3-4. https://doi.org/10.1038/s41416-020-0843-5

13. Cortiula F, Pettke A, Bartoletti M, Puglisi F, Helleday T. Managing COVID-19 in the oncology clinic and avoiding the distraction effect. Editorial Annals of Oncology Eur Soc Med Oncol. 2020;31(5):553-5. https://doi.org/10.1016/j. annonc.2020.03.286

14. Tan J, Yang C. Prevention and control strategies for the diagnosis and treatment of cancer patients during the COVID-19 pandemic. Brit J Cancer. 2020;123:5-6. http://doi. org/10.1038/s41416-020-0854-2

15. Facinal G, Oliveira VM. Breast cancer care during the coronavirus pandemic. Mastology. 2020;30:e20200014. http:// doi.org/10.29289/25945394202020200014

16. World Healh Organization. WHO list of priority medical devices for cancer management. Geneva: World Health Organization; 2017.

17. Instituto Nacional de Câncer José Alencar Gomes da Silva (INCA). Diretrizes para a detecção precoce do câncer de mama no Brasil. Rio de Janeiro: INCA; 2015.

18. Purcino FAC, Ruiz CA, Sorpreso ICE, Costa AMM, SoaresJunior JM, Baracat EC, et al. Management of benign and suspicious breast lesions during the coronavirus disease pandemic: recommendations for triage and treatment. Clinics. 2020;75:e2097. https://doi.org/10.6061/clinics/2020/e2097 